\title{
THE NORM MAP ON JACOBIANS
}

\author{
MICHAEL ROSEN
}

\begin{abstract}
Let $\pi: \Gamma \rightarrow \Gamma_{0}$ be an unramified normal cover of smooth projective curves. Let $\pi_{*}: J \rightarrow J_{0}$ be the induced map on Jacobians. Let $H$ be the kernel of $\pi_{*}$ and $H^{(0)}$ the connected component of $H$. We prove that $H / H^{()}$is isomorphic to $G /[G, G]$ where $G$ is the covering group of $\Gamma / \Gamma_{0}$.
\end{abstract}

Let $\Gamma$ and $\Gamma_{0}$ both be nonsingular, complete, connected curves defined over an algebraically closed field $K$. Let $J$ and $J_{0}$ be the Jacobians of $\Gamma$ and $\Gamma_{0}$ respectively. Finally, suppose $\pi: \Gamma \rightarrow \Gamma_{0}$ is a normal unramified covering and $\pi_{*}: J \rightarrow J_{0}, \pi^{*}: J_{0}$ $\rightarrow J$, the usual induced maps on Jacobians. The kernel of $\pi_{*}, H$, is an algebraic subgroup of $J$. Let $H^{0}$ denote the connected component of $H$. One can ask for the number of connected components of $H$. When $K=C$, the complex numbers, and $\pi: \Gamma \rightarrow \Gamma_{0}$ is a double cover, then it is classical that $\left[H: H^{0}\right]=2$. In this situation $H^{0}$ is called the Prym variety and its study is of great interest (see [2, Lecture IV]). Our main result is

THEOREM. If $G$ is the covering group of $\pi: \Gamma \rightarrow \Gamma_{0}$, then $H / H^{0} \approx G /[G, G]$.

The proof will be given in $\S 1$. The form of the result is reminiscent of class field theory. In fact, the well-known connection between abelian covers of $\Gamma_{0}$ and isogenies of $J_{0}$ can be exposited in a natural way using this theorem. We sketch this connection in $\$ 2$.

There are similarities between the material presented here and the work of $\mathrm{Y}$. Kawada and J. Tate in [1]. In particular, some of the cohomological calculations presented in $\S 1$ can be found there as well. For this reason we have not gone into great detail in some of the proofs.

We would like to thank Professor Joe Harris for raising the question of computing the index $\left[H: H^{0}\right]$.

1. Let $\mathscr{Q}, \mathcal{P}$, and $\mathcal{C}$ denote the divisors, principal divisors, and divisor classes on $\Gamma$. Let $J \subset \mathcal{C}$ be the divisor classes of degree zero. We identify $J$ with the Jacobian of $\Gamma$. All these groups are $G$ modules. Let $E=K(\Gamma)$ and $F=K\left(\Gamma_{0}\right)$ denote the function fields of $\Gamma$ and $\Gamma_{0}$ respectively. $E / F$ is a Galois extension and its Galois group may be identified with $G$. We begin by presenting a number of lemmas.

Received by the editors June 14, 1982. Presented to the Regional A.M.S. Meeting in Madison, Wisconsin, on April 17, 1982

1980 Mathematics Subject Classification. Primary 14H30, $14 \mathrm{H} 40$.

Key words and phrases. Jacobian, unramified cover, kernel of the norm map. 
LEMMA $1 . H^{-2}(G, \mathbf{Z}) \approx G /[G, G]$

LEMMA 2. VI) is cohomologically trivial.

LEMMA 3. $E^{*}$ is cohomologically trivial.

Proof. Lemma 1 is well known. Lemma 2 follows from the fact that in an unramified extension $\left.{ }^{\prime}\right)$ is $\mathbf{Z}[G]$ free.

To prove Lemma 3 it is sufficient to show $H^{i}\left(N, E^{*}\right)$ and $H^{i+1}\left(N, E^{*}\right)$ are trivial for some $i$ and all subgroups $N$ of $G$. Choose $i=1$. Then Hilbert's Theorem 90 and Tsen's theorem show what is required.

LemMA 4. $H^{-1}(G, \mathcal{U}) \approx \operatorname{Hom}\left(G, K^{*}\right)$.

Proof. From $(0) \rightarrow \mathscr{P} \rightarrow \mathscr{Q} \rightarrow \varrho \rightarrow(0)$ we conclude $H^{-1}(G, \varrho) \approx H^{0}(G, \mathscr{P})$ by Lemma 2.

From $(0) \rightarrow K^{*} \rightarrow E^{*} \rightarrow \varphi^{P} \rightarrow(0)$ we conclude $H^{0}(G, \mathscr{P}) \approx H^{1}\left(G, K^{*}\right)$ by Lemma 3.

Finally, since $K$ is fixed under $G, H^{1}\left(G, K^{*}\right) \approx \operatorname{Hom}\left(G, K^{*}\right)$.

LEMMA $5 . H^{-2}(G$, $)=(0)$.

Proof. Using the same arguments as in Lemma 4, we find $H^{-2}(G, \mathcal{C}) \approx$ $H^{-1}(G, ?) \approx H^{0}\left(G, K^{*}\right)=K^{*} / K^{* n}$ which is trivial since $K$ is algebraically closed. Here $n=|G|$.

Proposition 1. There is an exact sequence

$$
(0) \rightarrow G /[G, G] \stackrel{\alpha}{\rightarrow} H^{-1}(G, J) \stackrel{\beta}{\rightarrow} \operatorname{Hom}\left(G, K^{*}\right) \rightarrow(0) .
$$

Proof. From the exact sequence $(0) \rightarrow J \rightarrow \mathfrak{Z} \rightarrow \mathbf{Z} \rightarrow(0)$ we deduce

$$
H^{-2}(G, \varrho) \rightarrow H^{-2}(G, \mathbf{Z}) \rightarrow H^{-1}(G, J) \rightarrow H^{-1}(G, \mathcal{E}) .
$$

The last arrow is onto because $H^{-1}(G, \mathbf{Z})=(0)$. The result follows by Lemmas 5,1 , and 4.

Let $N=\sum \sigma \in \mathbf{Z}[G]$, the sum over all $\sigma \in G$, be the norm element. Let $I_{G} \subset \mathbf{Z}[G]$ be the augmentation ideal, i.e. the ideal generated by $\{\sigma-1 \mid \sigma \in G\}$. Then

$$
H^{-1}(G, J)={ }_{N} J / I_{G} J .
$$

We have used the notation ${ }_{N} J=\{j \in J \mid N j=0\}$.

By tracing through the definitions it is possible to describe the maps $\alpha$ and $\beta$ explicitly. Let $\gamma_{1} \in \Gamma$. Then for $\sigma \in G, \overline{\sigma \gamma_{1}-\gamma_{1}} \in{ }_{N} J$, where bar denotes the class of the divisor $\sigma \gamma_{1}-\gamma_{1}$. The map $\alpha$ is induced by the map $\sigma \rightarrow \overline{\sigma \gamma_{1}-\gamma_{1}}$. As must be the case, $\alpha$ does not depend on the choice of $\gamma_{1}$. As for $\beta$ we begin by noticing that an element of ${ }_{N} J / I_{G} J$ is represented by a divisor $Q$ such that $N Q=(\alpha)$ is principal. Since $N G^{\prime}$ is invariant under $G$ we must have $\sigma \alpha=\chi(\sigma) \alpha$ for all $\sigma \in G$ where $\chi(\sigma) \in K^{*}$. The map $\mathbb{Q} \rightarrow \chi$ induces $\beta$.

We need one more lemma.

LeMma 6. $I_{G} J=H^{0}$ (recall that $H^{0}$ is the connected component of $H=\operatorname{Ker} \pi_{*}$ ). 
Proof. As a mapping from $J \rightarrow J$ we have $N=\pi^{*} \pi_{*}$. Thus ker $\pi_{*}$ is contained in $\operatorname{ker} N$. Clearly $I_{G} J \subseteq \operatorname{ker} \pi_{*}$. By Proposition $1,{ }_{N} J / I_{G} J$ is finite. Thus it suffices to prove that $I_{G} J$ is connected.

Since $J$ is connected, so is $(\sigma-1) J$ for all $\sigma \in G$, and so is the product $\Pi(\sigma-1) J$. Map this product to $J$ by summing the coordinates. This is a continuous map, so the image is connected. The image is precisely $I_{G} J$.

We are now ready to prove the theorem. The proof will follow from Lemma 6 and Proposition 1 if we can show $\operatorname{ker} \beta=\operatorname{ker} \pi_{*} / I_{G} J$.

From its explicit description it is clear the image of $\alpha$ is contained in ker $\pi_{*} / I_{G} J$. Thus $\operatorname{ker} \beta \subseteq \operatorname{ker} \pi_{*} / I_{G} J$ and we must show that the reverse inclusion holds. Let $\mathbb{Q}$ be a divisor whose class represents an element of ker $\pi_{*} / I_{G} J$. Then $\pi_{*} Q=(a)$, a principal divisor on $\Gamma_{0}$. Thus $N Q=\pi^{*} \pi_{*} \mathbb{Q}=\pi^{*}(a)=\left(\pi^{*} a\right)$. Since $G$ fixes $\pi^{*} a$ we see that $\beta$ of the element of ker $\pi_{*} / I_{G} J$ corresponding to $Q$ is the trivial character. This establishes the reverse inclusion and completes the proof of the theorem.

2. We begin by recalling how isogenies of the Jacobian of $\Gamma_{0}$ give rise to abelian coverings of $\Gamma_{0}$.

Fix $\gamma_{0} \in \Gamma_{0}$ and map $\Gamma_{0}$ to $J_{0}$ by $\delta \rightarrow \overline{\delta-\gamma_{0}}$. Call this map $\iota_{0}$. Let $\lambda: A \rightarrow J_{0}$ be an isogeny, i.e. $A$ is an abelian variety, $\lambda$ is an onto homomorphism from $A$ to $J_{0}$ with finite kernel $N$. Form the fiber product $\Gamma_{0} \times_{J_{0}} A$. Recall that as a set this consists of all pairs $(\delta, a)$ such that $\iota_{0}(\delta)=\lambda(a)$. Projection onto the first coordinate makes $\Gamma_{0} \times J_{0} A$ into a normal covering of $\Gamma_{0}$ with covering group isomorphic to $N$. In fact, for $n \in N$ let $\sigma_{n}(\delta, \alpha)=(\delta, \alpha+n)$. Then $\sigma_{n}$ is a fiber preserving automorphism and $\left\{\sigma_{n} \mid n \in N\right\}$ is the whole covering group.

An important fact is that every unramified abelian covering of $\Gamma_{0}$ arises in just this way. We now indicate how our main theorem can be used to give a natural proof of this fact.

Let $\pi: \Gamma \rightarrow \Gamma_{0}$ be an unramified abelian covering with group $G$. Let $\gamma_{1} \in \Gamma$ and let $\gamma_{0}=\pi\left(\gamma_{1}\right) \in \Gamma_{0}$. Define $\iota: \Gamma \rightarrow J$ by $\iota(\gamma)=\overline{\gamma-\gamma_{1}}$ and $\iota_{0}: \Gamma_{0} \rightarrow J_{0}$ by $\iota_{0}(\delta)=\overline{\delta-\gamma_{0}}$. We then have the commutative square:

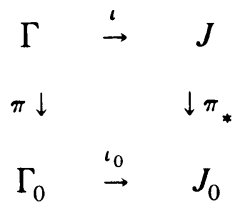

Let $A=J / H^{0}$ and $N=H / H^{0}$. Recall that the map $\alpha$ of Proposition 1 identifies $G$ with $N$.

Now, $A$ is an abelian variety and $\pi_{*}$ induces an isogeny $\lambda$ from $A$ to $J_{0}$ with kernel $N$.

The covering group of $\Gamma_{0} \times{ }_{J_{0}} A \rightarrow \Gamma_{0}$ can be identified with $G$ as follows; for $\sigma \in G, \sigma(\delta, a)=(\delta, a+\alpha(\sigma))$.

We define a map $f$ from $\Gamma$ to $\Gamma_{0} \times_{J_{0}} A$ as follows

$$
f(\gamma)=(\pi(\gamma), \overline{\iota(\gamma)})
$$

where the bar denotes the coset of $\iota(\gamma)$ modulo $H^{0}$. 
Proposition 2. The map $f$ is an isomorphism of $\Gamma$ with $\Gamma_{0} \times_{J_{0}} A$ as covering spaces of $\Gamma_{0}$.

Proof. $f$ clearly preserves fibers. Somewhat less clear is that $f$ is $G$-equivariant, i.e. $f(\sigma \gamma)=\sigma f(\gamma)$ for $\sigma \in G$ and $\gamma \in \Gamma$. To see this note $f(\sigma \gamma)=(\pi(\sigma \gamma), \overline{\iota(\sigma \gamma)})=$ $(\pi(\gamma), \overline{\iota(\sigma \gamma)})$. The second coordinate is by definition

$$
\overline{\sigma \gamma-\gamma_{1}}=\overline{\sigma \gamma-\sigma \gamma_{1}}+\overline{\sigma \gamma_{1}-\gamma_{1}}=\overline{\gamma-\gamma_{1}}+\alpha(\sigma) \text {. }
$$

Here we have used the definition of $\alpha$ and the fact that $(\sigma-1) \overline{\gamma-\gamma_{1}}=\overline{0}$ since $H^{0}=I_{G} J$. Since $\overline{\gamma-\gamma_{1}}=\overline{\imath(\gamma)}$ we have shown

$$
f(\sigma \gamma)=(\pi(\gamma), \iota(\gamma)+\alpha(\sigma))=\sigma(\pi(\gamma), \iota(\gamma))=\sigma f(\gamma) \text {. }
$$

The fact that $f$ is fiber preserving and $G$ equivariant together with the fact that $G$ is transitive on the fibers of both coverings implies easily that $f$ is onto. Since $G$ acts without fixed points it follows equally easily that $f$ is $1-1$. Thus $f$ is an isomorphism (in the sense of algebraic geometry) and the proof is complete.

\section{BIBLIOGRAPHY}

1. J. Kawada and J. Tate, On the Galois cohomology of unramified extensions of function fields in one variable, Amer. J. Math. 77 (1955), 197-217.

2. D. Mumford, Curves and their Jacobians, Univ. of Michigan Press, Ann Arbor, 1975.

Department of Mathematics, Brown University, Providence, Rhode Island 02906 